\title{
Computational Ways to Enhance Protein Inhibitor Design
}

\author{
Robert L. Jernigan ${ }^{1 *}$, Kannan Sankar ${ }^{1}, K_{\text {Kejue Jia }}{ }^{1}$, Eshel Faraggi ${ }^{2,3}$ and \\ Andrzej Kloczkowski ${ }^{4,5}$
}

${ }^{1}$ Roy J. Carver Department of Biochemistry, Biophysics and Molecular Biology, lowa State University, Ames, IA, United States, ${ }^{2}$ Research and Information Systems, LLC, Indianapolis, IN, United States, ${ }^{3}$ Department of Physics, Indiana University Purdue University Indianapolis, Indianapolis, IN, United States, ${ }^{4}$ Battelle Center for Mathematical Medicine, Nationwide Children's Hospital, Columbus, OH, United States, ${ }^{5}$ Department of Pediatrics, The Ohio State University, Columbus, $\mathrm{OH}$, United States

OPEN ACCESS

Edited by:

Hongchun Li,

Shenzhen Institutes of Advanced Technology (CAS), China

Reviewed by: Canan Atilgan,

Sabanci University, Turkey Igor N. Berezovsky, Bioinformatics Institute (A*STAR), Singapore

*Correspondence:

Robert L. Jernigan jernigan@iastate.edu

Specialty section:

This article was submitted to Biological Modeling and Simulation, a section of the journal

Frontiers in Molecular Biosciences

Received: 16 September 2020 Accepted: 08 December 2020 Published: 03 February 2021

Citation:

Jernigan RL, Sankar K, Jia K

Faraggi E and Kloczkowski A (2021)

Computational Ways to Enhance

Protein Inhibitor Design.

Front. Mol. Biosci. 7:607323. doi: 10.3389/fmolb.2020.607323
Two new computational approaches are described to aid in the design of new peptide-based drugs by evaluating ensembles of protein structures from their dynamics and through the assessing of structures using empirical contact potential. These approaches build on the concept that conformational variability can aid in the binding process and, for disordered proteins, can even facilitate the binding of more diverse ligands. This latter consideration indicates that such a design process should be less restrictive so that multiple inhibitors might be effective. The example chosen here focuses on proteins/peptides that bind to hemagglutinin (HA) to block the large-scale conformational change for activation. Variability in the conformations is considered from sets of experimental structures, or as an alternative, from their simple computed dynamics; the set of designe peptides/small proteins from the David Baker lab designed to bind to hemagglutinin, is the large set considered and is assessed with the new empirical contact potentials.

Keywords: protein design, peptide design, computational design, protein ensemble, protein potentials

\section{INTRODUCTION}

Influenza infection is a widespread cause of major medical concern because of rapid viral evolution, which causes both occasional pandemics and, more frequently, health problems almost every year. It has been estimated that the annual outbreaks by influenza A and B viruses over the past 100 years have had an even greater impact than all other past pandemics combined (Wilson et al., 1981; Bullough et al., 1994; Bizebard et al., 1995). The extremely high mutation rate of the virus means that any given vaccine soon becomes outdated. Thus, vaccination offers limited protection, especially when facing the highly virulent nature and rapid evolution of influenza (Chen et al., 1999). Although some effective anti-influenza drugs have been developed, drug resistance usually appears rapidly.

Hemagglutinin (HA) is a major surface glycoprotein of this virus that is involved in four of the most important aspects of influenza infection: (a) it is the target of antibodies that neutralize infectivity, (b) it undergoes antigenic drift to escape neutralization, (c) it binds to cell-surface receptors to initiate infection, and (d) it mediates the fusion of viral and host membranes essential for viral entry. The large-scale conformational changes in HA are critical for the steps in which the virus inserts itself into the host cells by fusing to the host membrane, and the residues involved in this process are highly conserved across different types and subtypes during antigenic drift. These 
residues can serve as important targets for developing broadreacting antiviral inhibitors (Jiang et al., 1993; Wild et al., 1994; Chan et al., 1998; Skehel and Wiley, 1998). Based on a set of crystal structures of the HA-antibody complex showing the conformational changes to HA during the essential activation steps, David Baker and his colleagues designed a novel HA inhibitor for Group 1 of type A virus (Fleishman et al., 2011).

Influenza $\mathrm{HA}$ is a homo-trimeric protein where each monomer contains two disulfide-bonded polypeptides, HA1 and HA2. HA1 is responsible for attaching to host cell-surface receptors, and HA2 mediates the fusion of the influenza envelope with the endosomal membrane thus allowing the entry of influenza RNA into the host cell. The pre- (Wilson et al., 1981) and post-fusion structures (Bizebard et al., 1995) of HA1 are essentially the same, while those of HA2 (Wilson et al., 1981; Bullough et al., 1994; Chen et al., 1999) are drastically different (see Figure 1).

The structural change in HA2 includes a partial unfolding of the long $\alpha$-helix into a loop (dark blue) and the folding of an inter-helix loop (in red) into a part of the long $\alpha$-helix, thus delivering both $\mathrm{N}$ - (blue) and C-terminal (pink) fragments to the same end of the molecule upon the fusion of viral and endosomal membranes.

The protein gp41 of HIV-1 is the membrane fusion protein, similar to HA2 of HA (Skehel and Wiley, 1998). In that case, peptides derived from the C-terminal region of gp41 corresponding to the outer-layer helices, referred to as Cpeptides, were found to inhibit HIV-1 infection with $\mathrm{IC}_{50}$ in the nanomolar range (Jiang et al., 1993; Wild et al., 1994; Chan et al., 1998). C-peptides are believed to act by binding to the

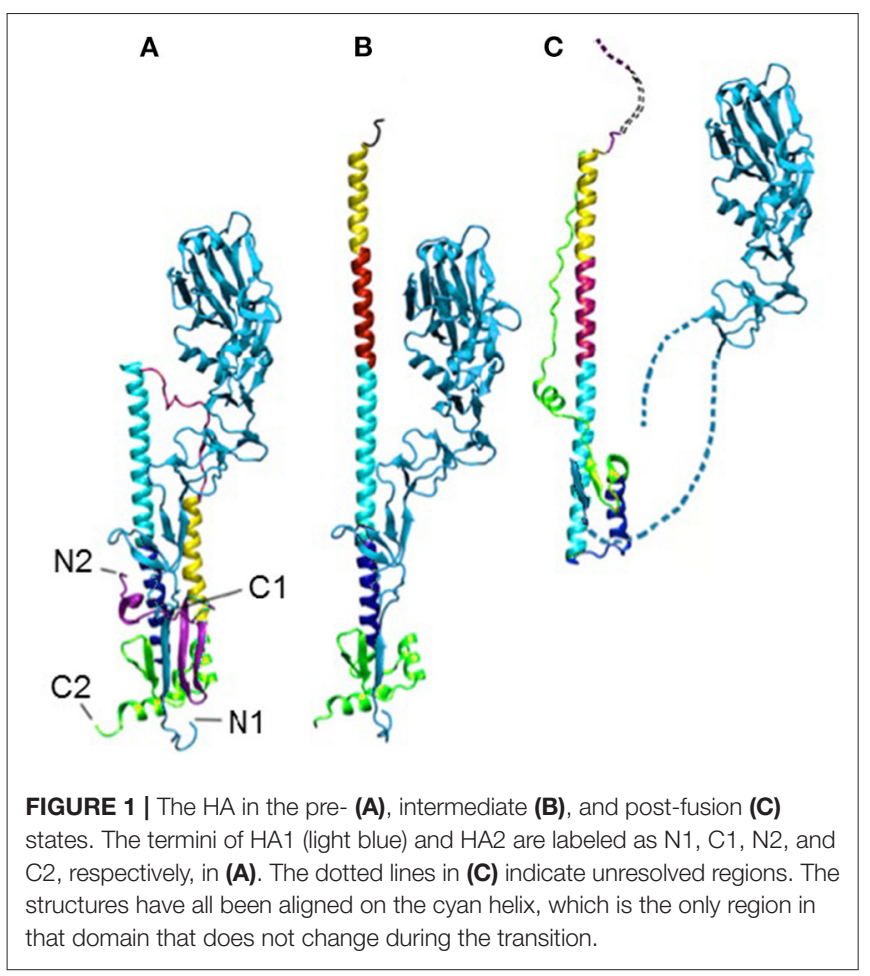

exposed surface of the N-terminal central three-helical bundle in a transient pre-fusion gp41 intermediate, thereby blocking membrane fusion. One such L-peptide, T-20/Enfuvirtide with 36 residues, was approved previously as a drug by the Food and Drug Administration (FDA) (FDA Notifications, 2003); it showed high efficacy in suppressing resistant HIV-1 strains. Moreover, efforts to target a prominent pocket on the surface of the central three-helical bundle have led to the discovery of small, cyclic D-peptides that inhibit HIV-1 infection, thereby validating the pocket as a potential target for small-molecule HIV-1 fusion inhibitors (Eckert et al., 1999).

To evade host antibody recognition, the HA protein on the surface of influenza virus, primarily on the globular domain, must constantly mutate. This interferes in important ways with any vaccine and reduces the vaccine's efficiency and useful lifetime. However, no matter how much the influenza virus mutates, it must maintain the ability to induce membrane fusion to ensure its propagation. Thus, the stem domain that is primarily responsible for inducing membrane fusion is the most conserved. Ian Wilson's group identified antibodies that broadly neutralize influenza A virus Group 1 (Ekiert et al., 2009) (Figure 2A), Group 2 (Murphy and Webster, 2001), Group 1 and 2 (Ekiert et al., 2012), and influenza type A and B viruses (Dreyfus et al., 2012) (see Figure 2B). All these antibodies recognize epitopes located in the stem domain. David Baker's group designed small proteins against influenza A virus Group 1 (Fleishman et al., 2011) (Figure 2A). In addition, they identified a conserved patch on the surface of the central helical bundle in the low-pH postfusion state (Figure 2C). These three interfaces may all serve as useful targets for developing inhibitors against influenza virus.

Molecular recognition in general and protein-protein interactions in particular are essential in almost every aspect of biological function. Moreover, proteins that bind other proteins with high affinity and high specificity have numerous applications for diagnostics and therapeutics. Currently, antibodies are by far the most commonly used proteins for both

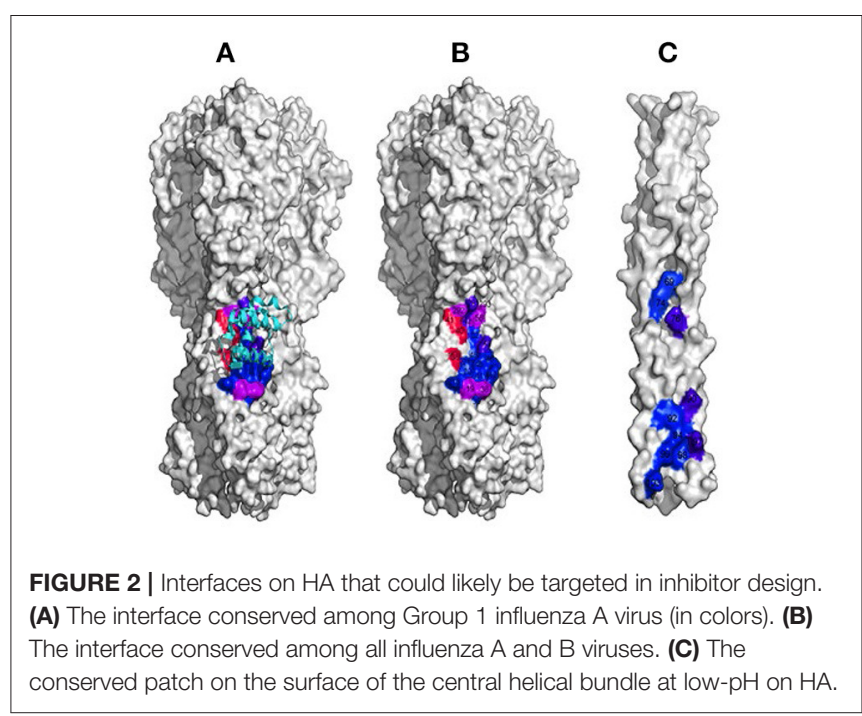


TABLE 1 | The PDB identifiers of the 43 structures of hemagglutinin used here for extracting dynamics.

\begin{tabular}{lllll}
\hline $1 \mathrm{HGD}$ & $2 \mathrm{HMG}$ & $3 \mathrm{FKU}$ & $4 \mathrm{BGZ}$ & $4 \mathrm{KPQ}$ \\
$1 \mathrm{MQL}$ & $2 \mathrm{BBX}$ & $3 \mathrm{HMG}$ & $4 \mathrm{BH} 1$ & $4 \mathrm{KPS}$ \\
$1 \mathrm{MQM}$ & $2 \mathrm{WR7}$ & $3 \mathrm{LZG}$ & $4 \mathrm{DJ} 6$ & $5 \mathrm{HMG}$ \\
$1 \mathrm{MQN}$ & $2 \mathrm{WRB}$ & $3 \mathrm{M} 5 \mathrm{G}$ & $4 \mathrm{EDB}$ & \\
$1 \mathrm{RD} 8$ & $2 \mathrm{WRD}$ & $3 \mathrm{M} 6 \mathrm{~S}$ & $4 \mathrm{~F} 23$ & \\
$1 \mathrm{RUY}$ & $2 \mathrm{WRE}$ & $3 \mathrm{~S} 11$ & $4 \mathrm{F3Z}$ & \\
$1 \mathrm{RUZ}$ & $2 \mathrm{WRF}$ & $3 \mathrm{SM} 5$ & $4 \mathrm{FIU}$ & \\
$1 \mathrm{RV0}$ & $2 \mathrm{WRG}$ & $3 \mathrm{UBE}$ & $4 \mathrm{GXX}$ & \\
$1 \mathrm{RVX}$ & $2 \mathrm{WRH}$ & $3 \mathrm{VUN}$ & $4 \mathrm{JTX}$ & \\
$2 \mathrm{FKO}$ & $3 \mathrm{EYM}$ & $3 Z \mathrm{TJ}$ & $4 \mathrm{KDM}$ & \\
\hline
\end{tabular}

detection and therapeutic intervention. However, antibodies are large proteins that are expensive to produce and difficult to deliver. Thus, it would be important progress for biomedicine to be able to design novel protein-binding modules at will.

The set of 88 proteins that were designed and tested by Baker et al. provides an excellent test set for use in the present study. Below we consider the dynamics of the structure in two different ways, from a set of experimental structures and from computed dynamics. Then we apply new knowledge-based free energies to rank the different designs, specifically predicting which designs are likely to bind. Baker and colleagues were not able to do this without experimental testing. These are empirical-free energy contact potentials developed by Jernigan, Kloczkowski, and Faraggi that have proven to be highly successfully in blind-tests at past CASP experiments. In the present paper, we aim to make some suggestions for new ways to sample conformations of a target protein and how to assess the designed structures.

\section{MULTIPLE EXPERIMENTAL STRUCTURES CAPTURE THE IMPORTANT FUNCTIONAL MOTIONS WITHIN A HEMAGGLUTININ STRUCTURE SET}

The 43 structures of hemagglutinin listed in Table 1 were collected from the PDB with a BLAST search, retaining only those structures present as trimeric complexes of the HA1 and HA2 subunits. The individual subunits were extracted separately and aligned. This yields a total of 129 structures of the HA1 + HA2 monomers that were superimposed onto the central structure (PDB: $1 \mathrm{mqm}$ ) using the Combinatorial Extension (CE) algorithm, and these have a continuous distribution of RMSDs from 0 to $3.3 \AA$.

After these structures have been superimposed, the covariances for all pairs of positions were computed. Then Principal Component Analysis is performed on this dataset. The input is the set of all of the structures in the set (Teodoro et al., 2002, 2003). From these data, the average position of each point in the reference structure is computed as $\left\langle x_{i}\right\rangle$, and the covariances for each pair of points, $i$ and $j$, was computed according to $c_{i j}=\left\langle\left(x_{i}-\left\langle x_{i}\right\rangle\right)\left(x_{j}-\left\langle x_{j}\right\rangle\right)\right\rangle$, where brackets $<>$ indicate averages over the set of structures. The covariance matrix

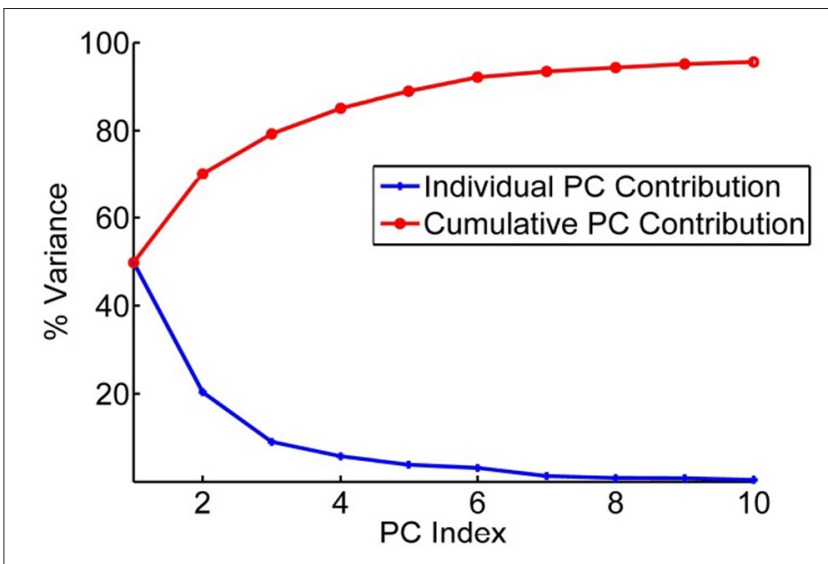

FIGURE 3 | Principal component contributions to the total motions of hemagglutinin. Percent of variance explained by each individual PC is shown in blue and the cumulative contribution of each PC to the total variance/motion in red. The first 5 PCs account for $90 \%$ of the total motions present in the set of 43 structures.

$C$ can be decomposed as $C=P \Delta P^{T}$, where the eigenvectors $P$ represent the principal components (PCs) and the eigenvalues are the elements of the diagonal matrix $\Delta$. The eigenvalues are sorted in order. Each eigenvalue is directly proportional to the amount of the total variance it captures. The results of this analysis are shown in Figure 3 for the set of coarse-grained hemagglutinins, which shows how truly limited the characteristic motions are within the structure set. Clearly, it does not require many of these characteristic motions to capture nearly all of the overall motions.

\section{CHARACTERIZATION OF THE GLOBAL MOTIONS IN HEMAGGLUTININS}

Based on their sequences, HAs have been subdivided into two main groups: Group 1 (H1, H2, H5, H6, H8, H9, H11, H12, H13, and H16) and Group 2 (H3, H4, H7, H10, H14, and H15) (Air, 1981). Interestingly, the first three PCs separately cluster into these two major groups, with minor exceptions. The distribution of the experimental structures over the PCs are shown in Figure 4 for pairs of PCs. This distinctive clustering can be seen clearly.

Different conformations can bind to different partners and thus include dynamics in the process that will improve the probability of success in computational protein design. When the PCs are visualized on the structures, it can be seen that the first three PCs primarily represent motions in the B-loop (blue) that are involved in the large-scale transition. PC1, PC2, and PC3 can be interpreted as primarily involving conformations changes in the C-terminus, the central, and N-terminus parts of the Bloop (see Figure 5). Interestingly, the B-loop is a region with a strong tendency to form a coiled-coil and is implicated in the formation of the pre-hairpin intermediate in the "spring loaded mechanism" of HA action (Carr and Kim, 1993; Xu and Wilson, 2011). The PC3 motion also clearly demonstrates the shift in the loop necessary for it to position itself at the top of helix C. In 

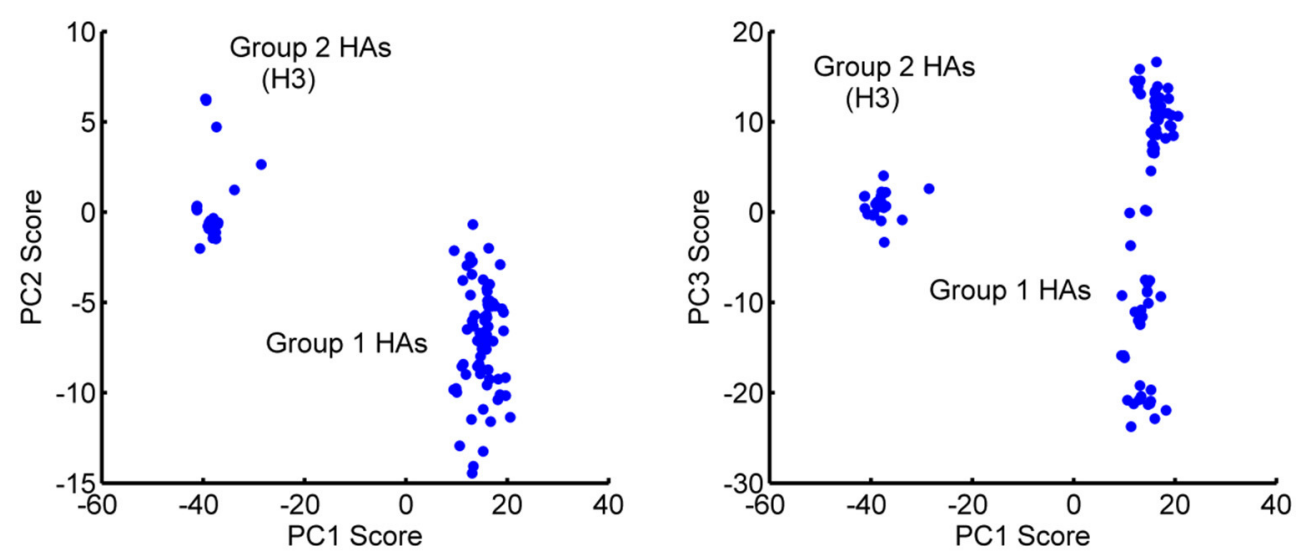

FIGURE 4 | Distribution of the 129 HA monomeric structures projected onto the first 3 PCs. (Left) PC2-PC1 space and (Right) PC3-PC1 space. PC1 separates the Group 1 and Group 2 hemagglutinins into different clusters. Outliers $\mathrm{H} 13$ and $\mathrm{H} 16$ have been eliminated from the figure for the sake of clarity. PC1 has a major gap between the two groups of clustered structures. PC3 appears to be populated in two somewhat similar clusters, with Group 1 showing a particularly wide range of PC3 values.

addition, PC2 captures a hinge motion in the head of HA with respect to the stem as well as well as a motion at the $\mathrm{N}$-terminus of HA2 (fusion peptide) that is subsequently exposed for insertion into the membrane during fusion. These computed structures show a high level of variability of conformations particularly for the B-loop, which relate well to the known conformational transition, even though the full extent of motions is not shown in Figure 5. As shown in Table 2, these PCs provide a useful representation of changes present in the ensemble of structures.

\section{ANISOTROPIC NETWORK MODELS (ANM) CAN SUBSTITUTE, OF INSUFFICIENT NUMBERS OF EXPERIMENTAL STRUCTURES ARE AVAILABLE}

Elastic Network Models of proteins, such as the Gaussian Network Model (GNM) and Anisotropic Network Models (ANM) of proteins as developed by Tirion (1996), Bahar, Erman, and Jernigan (Bahar and Jernigan, 1994, 1998; Bahar et al., 1997a; Demirel et al., 1998; Atilgan et al., 2001; Doruker et al., 2002a,b; Doruker and Jernigan, 2003; Sen et al., 2006), computationally yield information about protein fluctuation dynamics, the directions of motions of the residues and atoms around their equilibrium positions. This information has already been used by Bahar, Jernigan, Kloczkowski, and many others with significant success (Bahar and Jernigan, 1994; Keskin et al., 2002a,b; Isin et al., 2012) to explain functional motions and mechanisms in proteins, nucleic acids, and large biological assemblies, such as the ribosome. ANM could be used as an alternative to calculate the normal modes from a single structure when insufficient numbers of experimental structures or structures having sufficient variability are not available to perform PC analysis, then normal modes from the elastic network models could also be used to compute entropies (Zimmermann et al., 2012) (But, as we show below, contact entropies are simpler and provide significant gains). In ANM, the potential energy $V$ is a function of the displacement vector $D$ of each point in the structure $V=\frac{\gamma}{2} D H D^{T}$, where $\gamma$ is the spring constant for all closely interacting points in a structure (here we used a cutoff distance of $13 \AA$ between alpha-carbons for coarsegrained models retaining only $\mathrm{C}^{\alpha}$ atoms) to establish the spring connections between residues), and $H$ is the Hessian matrix containing the second derivatives of the energy, with respect to each of the coordinates $\mathrm{x}, \mathrm{y}, \mathrm{z}$. For a structure with $n$ residues, the Hessian matrix $H$ contains $n \times n$ super-elements each of size $3 \times 3$. The Hessian matrix $H$ can be decomposed (Atilgan et al., 2001) as $H=M \Lambda M^{T}$, where $\Lambda$ is a diagonal matrix comprising the eigenvalues with the eigenvectors forming the columns of the matrix $M$. This decomposition generates $3 n-6$ normal modes (the first six modes account for the rigid body translations and rotations of the system) reflecting the vibrational fluctuations, so singular value decomposition is utilized.

\section{COMPARING DIRECTIONS OF MOTIONS USING OVERLAPS}

The alignment between the directions of a given experimental PC and a given computed normal mode can be measured by comparing the directions of motion in their overlap, as defined by Tama and Sanejouand (2001): $O_{i j}=\frac{\left|P_{i} \cdot M_{j}\right|}{\left\|P_{i}\right\|\left\|M_{j}\right\|}$, where $P_{i}$ is the $i$ th PC for and $M_{j}$ is the $j$ th normal mode. A perfect match yields an overlap value of 1 , meaning these motions are in the same direction. We also define the cumulative overlap (CO) between the first $k$ vectors $M_{\mathrm{j}}$ and $P_{i}$ as $C O(k)=\left(\sum_{j=1}^{k} O_{i j}^{2}\right)^{\frac{1}{2}}$.

The high overlaps between the two methods ensures the reliability of the computed dynamics. The 1st, 2nd, and 3rd PCs have good overlaps of $0.57,0.43$, and 0.34 with the $3 \mathrm{rd}, 2 \mathrm{nd}$, and 1st individual modes, respectively. We compare the first three 


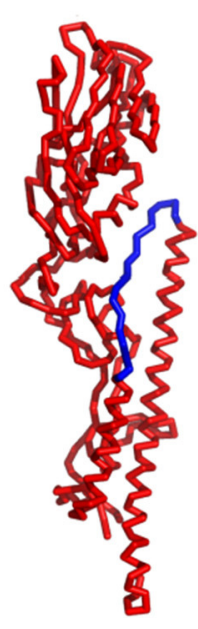

PC1

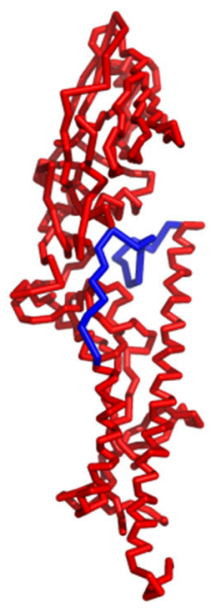

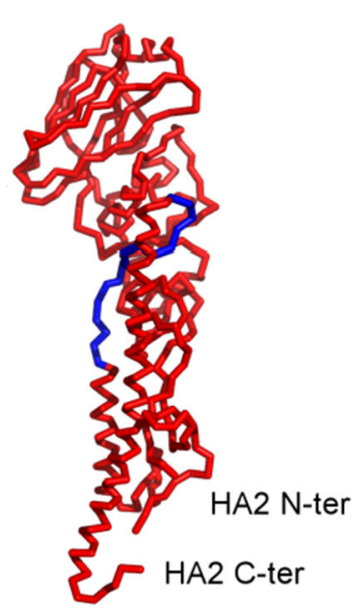

PC2

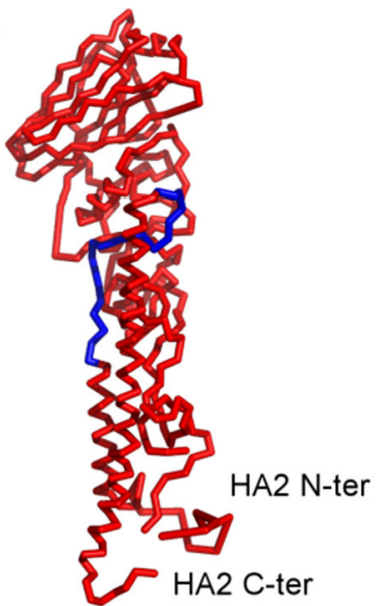

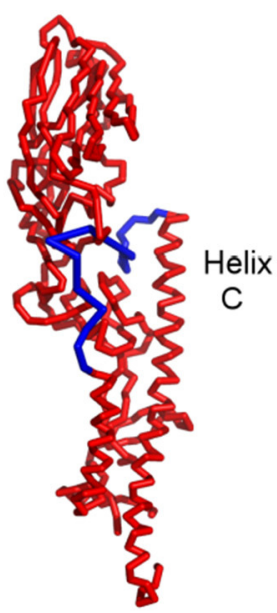

PC3

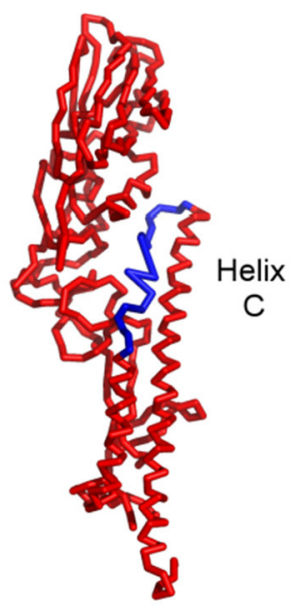

FIGURE 5 | Visualization of the first three PC motions on the structures of HA. The two structures shown in each column are two extreme conformations representative of the changes indicated in each individual PC. PCs 1, 2, and 3 can be identified as winding and unwinding of the C-terminal, central, and N-terminal parts of the B-loop (blue) into a helix. PC2 captures the hinge bending of the structure between the head and stem regions as well as movement of the N-terminus of HA2. The blue highlighted segments indicate the parts of the structure exhibiting a broad range of conformations.

PC's from the X-ray set with the first 20 normal modes from the elastic network models, and these are relatively high between all three PCs of the X-ray hemagglutinin and the set of normal modes for the computed normal modes (see Table 2).

\section{STRATEGIES FOR GENERATING AND RANKING AN ENSEMBLE OF STRUCTURES AND IDENTIFYING A STRUCTURE MODULE TARGETED FOR INHIBITOR DESIGN}

Identifying the most conformationally variable part of the structure is the aim here. These are the parts of a structure that should be the most useful to use for inhibitor design. These parts can be identified simply by computing the changes in all internal
TABLE 2 | Cumulative overlaps between computed ANM modes and PCs from the set of experimental hemagglutinin structures.

\begin{tabular}{lccc}
\hline & \multicolumn{3}{c}{ co } \\
\cline { 2 - 4 } & 3 Modes & 6 Modes & 20 Modes \\
\hline PC1 & 0.60 & $\mathbf{0 . 6 6}$ & $\mathbf{0 . 7 1}$ \\
PC2 & $\mathbf{0 . 5 0}$ & $\mathbf{0 . 5 7}$ & $\mathbf{0 . 6 5}$ \\
PC3 & 0.40 & 0.44 & $\mathbf{0 . 6 0}$
\end{tabular}

Values above 0.5 are in bold.

distances over the ensemble. Examples of such potential binding parts to target have been extracted from the ensemble of sampled conformations for HA generated by utilizing combinations of the first several PCs (Figure 6). This highly variable segment should be susceptible to binding by a broader range of ligands. 

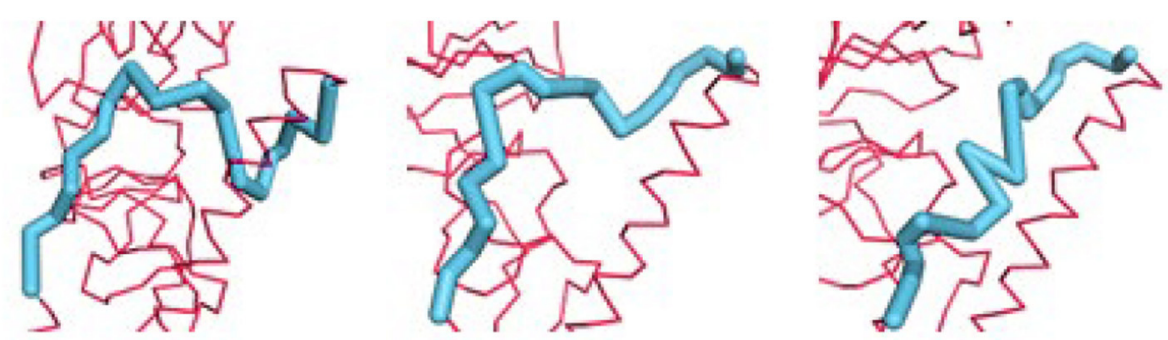

FIGURE 6 | Examples of the diversity of conformations from the first 3 PCs for the B loop (blue) of hemagglutinin. The PCs can be used to generate an ensemble of conformations. Each of three shows a conformation generated from one PC. The motions showed that this loop is the most flexible part of the structure and possesses an extremely diverse set of conformations.

\section{ASSESSING PEPTIDE/PROTEIN DESIGNS WITH NEW EMPIRICAL CONTACT POTENTIALS}

Here we present new strategies for the assessment of bound ligand structures by taking as our target the designed small proteins from David Baker and his colleagues that were targeted to bind hemagglutinin (Fleishman et al., 2011; Fleishman and Baker, 2012). This provides an interesting relatively large dataset, which we can use to test our assessment method. The Baker designs, originating from small, monomeric proteins in the PDB having between 80 and 250 residues, were targeted against a hydrophobic region on the "stem" of hemagglutinin. Of the 88 designs that they tested, only two were reported to have detectable binding affinity for hemagglutinin (this affinity was subsequently improved in rounds of randomization and selection).

\section{Four-Body Coarse-Grained Contact Potentials (Feng et al., 2007, 2010)}

Four-body potentials were developed by Kloczkowski and Jernigan to account for the cooperative interactions in proteins; they take into account the coarse-grained contact interactions together with the extent of solvent exposure, and thus provide a more detailed and more cooperative representation of protein interaction energies than do pairwise potentials. Capturing this cooperativity is considered to be critical for evaluating densely packed protein structures. These potentials are highly empirical and are based simply on the observed frequency of occurrences of different types of amino acids in closely interacting quartets of amino acid types within a large set of protein structures. We have found that these four-body contact potentials can discriminate well between native structures and partially unfolded or deliberately misfolded structures. These have also included short-range backbone energies (Bahar et al., 1997b). We tested these optimized potentials at CASP9 as the prediction group 4_BODY_POTENTIALS from Iowa State University. There were 110 other human prediction groups participating in CASP9 competition, and 140 prediction servers. According to Nick Grishin, the assessor of free modeling techniques at CASP9, 4_BODY_POTENTIALS was one of most successful groups in free modeling at that time, ranking third according to the averaged $\mathrm{z}_{\text {score }}$ both for best models and top models. Free modeling is the most difficult and most challenge in protein structure prediction, when the sequence of the protein has only a low sequence similarity in comparison to any known protein structures. This success at CASP9 demonstrates clearly that the cooperative multibody interactions are an appropriate tool for assessing predicted structures, and we apply them here to Baker's hemagglutinin inhibitor structures. Later we added in electrostatic interactions, and these were tested at the subsequent CASP10.

\section{Including Entropies in the Inhibitor Assessments}

The Elastic Network Models (ENM) have proven themselves to be highly useful in representing the global motions for a wide variety of diverse protein structures (Bahar and Jernigan, 1997, 1998, 1999; Bahar et al., 1997a,b,c; Bahar et al., 1998, 1999; Demirel et al., 1998; Keskin et al., 1998, 2000, 2002a,b; Jernigan et al., 1999, 2000, 2008; Atilgan et al., 2001; Bahar and Rader, 2005; Sen et al., 2006; Jernigan and Kloczkowski, 2007; Yang et al., 2007, 2008, 2009; Zhu and Hummer, 2010; Bakan et al., 2011; Karaca and Bonvin, 2011; May and Brooks, 2011; Peng and Head-Gordon, 2011; Uyar et al., 2011; Wieninger et al., 2011; Zheng, 2011; Zheng and Auerbach, 2011; Zimmermann et al., 2011a,b; Duttmann et al., 2012; Gniewek et al., 2012; Isin et al., 2012; Martin et al., 2012; Ruvinsky et al., 2012; Globisch et al., 2013; Kim et al., 2013; Sanejouand, 2013; Dasgupta et al., 2014). Since they have proven to be so successful in capturing the most important motions of protein structures, it is reasonable to expect that they should also be able to estimate the conformational entropies of structures. We employ the Elastic Network Model to compute the motions of protein structures, and then these motions are then used directly to approximate the entropy of a conformation (Zimmermann et al., 2011c, 2012). We previously (Zimmermann et al., 2011c) used vibrational entropies based on the frequencies of the normal modes, but more recently have found significant gains by utilizing the mean square fluctuations computed from the ENM as a direct measure of entropy: $\Delta S \propto$ 


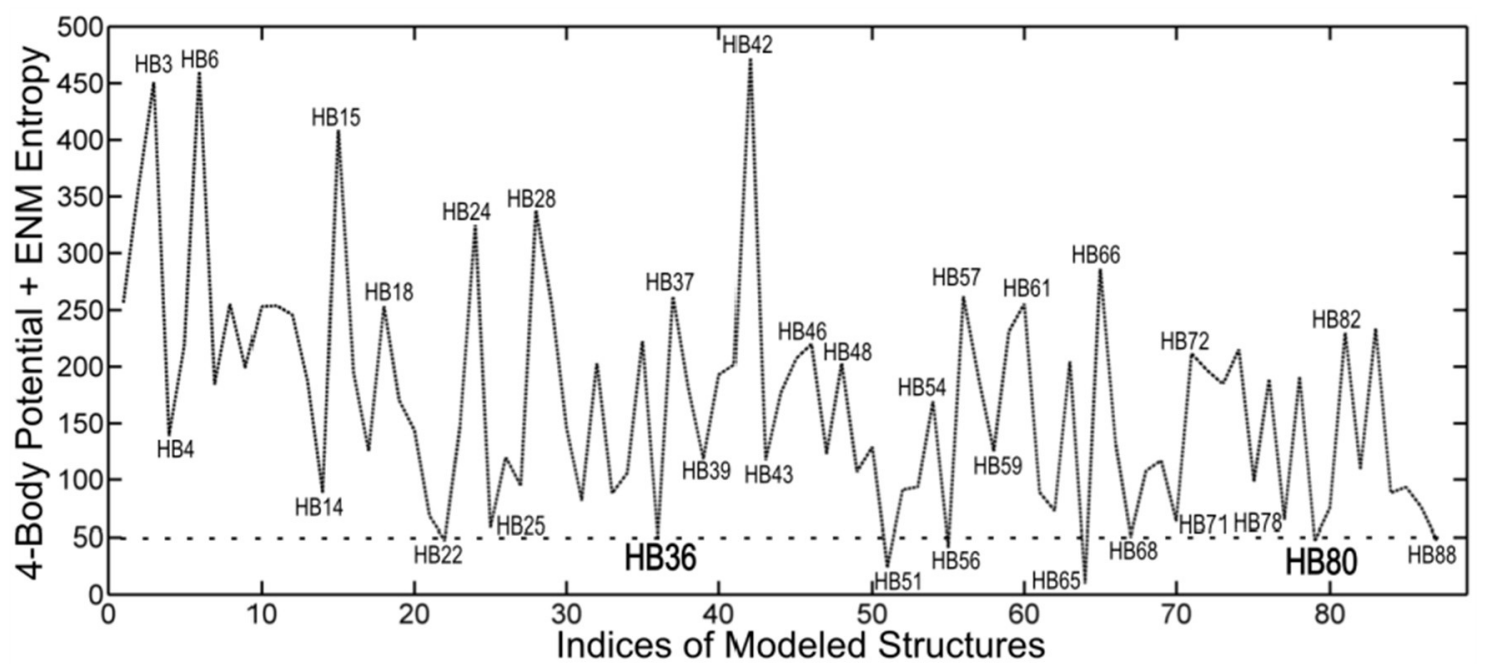

FIGURE 7 | Ranking by coarse-grained free energies of inhibitor proteins designed against hemagglutinin. Free energies are given on the ordinate axis (arbitrary scale), and the different structures (from the pdb) are indicated along the abscissa. The eight top-ranked structures with favorable free energies can be seen to be HB22, HB36, HB51, HB56, HB65, HB68, HB80, and HB88. This demonstrates the utility of the coarse-grained free energies to computationally screen for favorable structures. The two structures HB36 and HB80 were experimentally shown to be functional.

$\Gamma^{-1}=\sum_{i=2}^{N} \frac{1}{\lambda_{i}}\left(Q_{i} Q_{i}^{T}\right)$, where $Q$ is a normal mode vector, $\lambda$ the corresponding square frequency, $\Gamma$ the system's Hessian, and $\Gamma^{-1}$ its pseudo-inverse. We obtain the Free Energy changes from $\Delta \mathrm{G}=\Delta \mathrm{E}-\mathrm{T} \Delta \mathrm{S}$ by simply combining the four-body potential with the ENM-based entropy (Zimmermann et al., 2012). The excellent blind-tested performance of our method in CASP experiments shows that our methodology is an outstanding tool for assessing protein designs, such as the ones from Baker's hemagglutinin inhibitor designs.

\section{THESE NEW FREE ENERGIES SUCCESSFULLY SELECT NATIVE-LIKE POSES IN PROTEIN-PROTEIN DOCKING}

We have applied this method to the set of 89 inhibitor proteins designed against hemagglutinin by David Baker's group, and we find that it provides a useful screen for that set of structures. Structures having the lowest energies indicate stable favorable conformations. However, stable structures are not always functional. In this case, we tested a set of eight structures at local minimums of the energy landscape ranked by their energies. From these, it was reported that two of them were found to be functional (see Figure 7).

\section{DISCUSSION}

We have outlined a simple new way to use protein dynamics for peptide/protein design studies. This approach serves to identify those specific regions in the structure having particularly wide-ranging conformational variability, which could be of particular importance for targeting computational design efforts. Specifically, the highly variable segments should be able to bind to a particularly wide range of diverse ligands. Such variable conformations are well-known to be important for the promiscuous binding exhibited by disordered proteins and using this approach should have some advantage. Using such more localized protein targets might be an important new approach for targeted computational design. Another advantage of this is that more exhaustive computations can be carried out for smaller targets.

Application of the potentials described above to assess structural designs would allow ranking of sets of designed inhibitor proteins. The differences in rankings should allow to conclude the extent to which the large-scale backbone fluctuations identified in the dynamics could be utilized in the design process. This would require a significantly larger effort than has been presented here. Of course, the potentials themselves are empirical and could be modified to reflect the data from the experimental studies on the designed molecules for the specific class of targeted protein, which is one of the major advantages of the adaptability of the empirical potentials in any particular application.

Our approach can be extended by detailed analysis of allosteric sites that are important for drug design. Most drugs are designed to bind directly to the primary active sites, called orthosteric sites, to inhibit or modify the function of the protein. Binding of a drug to the active site prevents binding to a virus or other diseaserelated agent and most drugs are designed to fit into the primary active sites. However, adverse side effects of a drug may occur because many enzymes or receptors with related functions may have similarities in their active sites.

A new approach to drug design is based on secondary binding site effects. In this approach, small molecule drugs are designed to bind at secondary binding sites called allosteric sites (Tsai and Nussinov, 2014; Dokholyan, 2016; Guarnera and Berezovsky, 2016, 2020; Schueler-Furman and Wodak, 2016; Wodak et al., 
2019; Zhang et al., 2020). A potential drug-an allosteric modulator binds to an allosteric site and remotely modifies the conformation of the primary binding site of the protein. Allosteric sites are controlled by intrinsic protein dynamics, and the approach proposed here could also be applied to these allosteric sites.

\section{DATA AVAILABILITY STATEMENT}

Publicly available datasets were analyzed in this study. This data can be found at: protein data bank.

\section{REFERENCES}

Air, G. M. (1981). Sequence relationships among the hemagglutinin genes of 12 subtypes of influenza A virus. Proc. Natl. Acad. Sci. U.S.A. 78, 7639-7643. doi: $10.1073 /$ pnas.78.12.7639

Atilgan, A. R., Durell, S. R., Jernigan, R. L., Demirel, M. C., Keskin, O., and Bahar, I. (2001). Anisotropy of fluctuation dynamics of proteins with an elastic network model. Biophys. J. 80, 505-515. doi: 10.1016/S0006-3495(01)76033-X

Bahar, I., Atilgan, A. R., and Erman, B. (1997a). Direct evaluation of thermal fluctuations in proteins using a single-parameter harmonic potential. Fold. Des. 2, 173-181. doi: 10.1016/S1359-0278(97)00024-2

Bahar, I., Erman, B., Haliloglu, T., and Jernigan, R. L. (1997c). Efficient characterization of collective motions and interresidue correlations in proteins by low-resolution simulations. Biochemistry 36, 13512-13523. doi: $10.1021 /$ bi971611f

Bahar, I., Erman, B., Jernigan, R. L., Atilgan, A. R., and Covell, D. G. (1999). Collective motions in HIV-1 reverse transcriptase: examination of flexibility and enzyme function. J. Mol. Biol. 285, 1023-1037. doi: 10.1006/jmbi.1998.2371

Bahar, I., and Jernigan, R. L. (1994). Cooperative structural transitions induced by non-homogeneous intramolecular interactions in compact globular proteins. Biophys. J. 66, 467-481. doi: 10.1016/S0006-3495(94)80798-2

Bahar, I., and Jernigan, R. L. (1997). Inter-residue potentials in globular proteins and the dominance of highly specific hydrophilic interactions at close separation. J. Mol. Biol. 26, 195-214. doi: 10.1006/jmbi.1996.0758

Bahar, I., and Jernigan, R. L. (1998). Vibrational dynamics of transfer RNAs: comparison of the free and synthetase-bound forms. J. Mol. Biol. 281, 2522-2532. doi: 10.1006/jmbi.1998.1978

Bahar, I., and Jernigan, R. L. (1999). Cooperative fluctuations and subunit communication in tryptophan synthase. Biochemistry 38, 3478-3490. doi: $10.1021 / \mathrm{bi} 982697 \mathrm{v}$

Bahar, I., Kaplan, M., and Jernigan, R. L. (1997b). Short-range conformational energies, secondary structure propensities, and recognition of correct sequence-structure matches. Proteins 29, 292-308. doi: 10.1002/(SICI)10970134(199711)29:3<292::AID-PROT4>3.0.CO;2-D

Bahar, I., and Rader, A. J. (2005). Coarse-grained normal mode analysis in structural biology. Curr. Opin. Struct. Biol. 15, 586-592. doi: 10.1016/j.sbi.2005.08.007

Bahar, I., Wallqvist, A., Covell, D. G., and Jernigan, R. L. (1998). Correlation between native-state hydrogen exchange and cooperative residue fluctuations from a simple model. Biochemistry 37, 1067-1075. doi: 10.1021/bi9720641

Bakan, A., Meireles, L. M., and Bahar, I. (2011). ProDy: protein dynamics inferred from theory and experiments. Bioinformatics 27, 1575-1577. doi: 10.1093/bioinformatics/btr168

Bizebard, T., Gigant, B., Rigolet, P., Rasmussen, B., Diat, O., Bosecke, P., et al. (1995). Structure of influenza virus haemagglutinin complexed with a neutralizing antibody. Nature 376, 92-94. doi: 10.1038/37 6092a0

Bullough, P. A., Hughson, F. M., Skehel, J. J., and Wiley, D. C. (1994). Structure of influenza haemagglutinin at the $\mathrm{pH}$ of membrane fusion. Nature 371, 37-43. doi: $10.1038 / 371037 \mathrm{a} 0$

\section{AUTHOR CONTRIBUTIONS}

RJ, KS, KJ, and AK conceptualized, carried out the research, and wrote the paper. EF engaged in discussions about this research. All authors contributed to the article and approved the submitted version.

\section{FUNDING}

This research was supported by NSF grant DBI-1661391 and NIH grant R01GM127701.

Carr, C. M., and Kim, P. S. (1993). A spring-loaded mechanism for the conformational change of influenza hemagglutinin. Cell 73, 823-832. doi: 10.1016/0092-8674(93)90260-W

Chan, D. C., Chutkowski, C. T., and Kim, P. S. (1998). Evidence that a prominent cavity in the coiled coil of HIV type 1 gp41 is an attractive drug target. Proc. Natl. Acad. Sci. U.S.A. 95, 15613-15617. doi: 10.1073/pnas.95.26.15613

Chen, J., Skehel, J. J., and Wiley, D. C. (1999). N- and C-terminal residues combine in the fusion-pH influenza hemagglutinin $\mathrm{HA}(2)$ subunit to form an $\mathrm{N}$ cap that terminates the triple-stranded coiled coil. Proc. Natl. Acad. Sci. U.S.A. 96, 8967-8972. doi: 10.1073/pnas.96.16.8967

Dasgupta, B., Nakamura, H., and Kinjo, A. R. (2014). Rigid-body motions of interacting proteins dominate multispecific binding of ubiquitin in a shapedependent manner. Proteins 82, 77-89. doi: 10.1002/prot.24371

Demirel, M. C., Atilgan, A. R., Jernigan, R. L., Erman, B., and Bahar, I. (1998). Identificatioin of kineticallyl hot residues in proteins. Protein Sci. 7, 871-884. doi: $10.1002 /$ pro.5560071205

Dokholyan, N. V. (2016). Controlling allosteric networks in proteins. Chem. Rev. 116, 6463-6487. doi: 10.1021/acs.chemrev.5b00544

Doruker, P., and Jernigan, R. L. (2003). Functional motions can be extracted from on-lattice construction of protein structures. Proteins 53, 174-181. doi: 10.1002/prot.10486

Doruker, P., Jernigan, R. L., and Bahar, I. (2002a). Dynamics of large proteins through hierarchical levels of coarse-grained structures. J. Comput. Chem. 23, 119-127. doi: 10.1002/jcc. 1160

Doruker, P., Jernigan, R. L., Navizet, I., and Hernandez, R. (2002b). Important fluctuation dynamics of large protein structures are preserved upon renormalization. Int. J. Quantum Chem. 90, 822-837. doi: 10.1002/qua.955

Dreyfus, C., Laursen, N. S., Kwaks, T., Zuijdgeest, D., Khayat, R., Ekiert, D. C., et al. (2012). Highly conserved protective epitopes on influenza B viruses. Science 337, 1343-1348. doi: 10.1126/science.1222908

Duttmann, M., Mittnenzweig, M., Togashi, Y., Yanagida, T., and Mikhailov, A. S. (2012). Complex intramolecular mechanics of G-actin-an elastic network study. PLoS ONE 7:e45859. doi: 10.1371/journal.pone.0045859

Eckert, D. M., Malashkevich, V. N., Hong, L. H., Carr, P. A., and Kim, P. S. (1999). Inhibiting HIV-1 entry: discovery of D-peptide inhibitors that target the gp41 coiled-coil pocket. Cell 99, 103-115. doi: 10.1016/S0092-8674(00)80066-5

Ekiert, D. C., Bhabha, G., Elsliger, M. A., Friesen, R. H., Jongeneelen, M., Throsby, M., et al. (2009). Antibody recognition of a highly conserved influenza virus epitope. Science 324, 246-251. doi: 10.1126/science.1171491

Ekiert, D. C., Kashyap, A. K., Steel, J., Rubrum, A., Bhabha, G., Khayat, R., et al. (2012). Cross-neutralization of influenza A viruses mediated by a single antibody loop. Nature 489, 526-532. doi: 10.1038/nature11414

FDA Notifications (2003). FDA Approves Fuzeon, The First Fusion Inhibitor. Rockville, MD: AIDS Alert 18.

Feng, Y., Kloczkowski, A., and Jernigan, R. L. (2007). Four-body contact potentials derived from two protein datasets to discriminate native structures from decoys. Proteins 68, 57-66. doi: 10.1002/prot.21362

Feng, Y., Kloczkowski, A., and Jernigan, R. L. (2010). Potentials 'R' Us webserver for protein energy estimations with coarse-grained knowledge-based potentials. BMC Bioinformatics 11:92. doi: 10.1186/1471-2105-11-92 
Fleishman, S. J., and Baker, D. (2012). Role of the biomolecular energy gap in protein design, structure, and evolution. Cell 149, 262-273. doi: 10.1016/j.cell.2012.03.016

Fleishman, S. J., Whitehead, T. A., Ekiert, D. C., Dreyfus, C., Corn, J. E., Strauch, E. M., et al. (2011). Computational design of proteins targeting the conserved stem region of influenza hemagglutinin. Science 332, 816-821. doi: $10.1126 /$ science. 1202617

Globisch, C., Krishnamani, V., Deserno, M., and Peter, C. (2013). Optimization of an elastic network augmented coarse grained model to study CCMV capsid deformation. PLoS ONE 8:e60582. doi: 10.1371/journal.pone.0060582

Gniewek, P., Kolinski, A., Jernigan, R. L., and Kloczkowski, A. (2012). Elastic network normal modes provide a basis for protein structure refinement. $J$. Chem. Phys. 136:195101. doi: 10.1063/1.4710986

Guarnera, E., and Berezovsky, I. N. (2016). Allosteric sites: remote control in regulation of protein activity. Curr. Opin. Struct. Biol. 37, 1-8. doi: 10.1016/j.sbi.2015.10.004

Guarnera, E., and Berezovsky, I. N. (2020). Allosteric drugs and mutations: chances, challenges, and necessity. Curr. Opin. Struct. Biol. 62, 149-157. doi: $10.1016 /$ j.sbi.2020.01.010

Isin, B., Tirupula, K. C., Oltvai, Z. N., Klein-Seetharaman, J., and Bahar, I. (2012). Identification of motions in membrane proteins by elastic network models and their experimental validation. Methods Mol. Biol. 914, 285-317. doi: 10.1007/978-1-62703-023-6_17

Jernigan, R. L., Bahar, I., Covell, D. G., Atilgan, A. R., Erman, B., and Flatow, D. T. (2000). Relating the structure of HIV-1 reverse transcriptase to its processing step. J. Biomol. Struct. Dyn. 17, 49-55. doi: 10.1080/07391102.2000.10506603

Jernigan, R. L., Demirel, M. C., and Bahar, I. (1999). Relating structure to function through the dominant modes of motion of DNA topoisomerase II. Int. J. Quant. Chem. 175, 301-312. doi: 10.1002/(SICI)1097-461X(1999)75:3<301::AIDQUA19>3.0.CO;2-0

Jernigan, R. L., and Kloczkowski, A. (2007). Packing regularities in biological structures relate to their dynamics. Methods Mol. Biol. 350, 251-76. doi: 10.1385/1-59745-189-4:251

Jernigan, R. L., Yang, L., Song, G., and Doruker, P. (2008). "Elastic network models of coarse-grained proteins are effective for studying the structural control exerted over their dynamics," in Coarse-Graining of Condensed Phase and Biomolecular Systems, ed G. Voth (Taylor and Francis Group LLC; CRC Press), 237-254. doi: 10.1201/9781420059564.ch16

Jiang, S., Lin, K., Strick, N., and Neurath, A. R. (1993). HIV-1 inhibition by a peptide. Nature 365:113. doi: 10.1038/365113a0

Karaca, E., and Bonvin, A. M. (2011). A multidomain flexible docking approach to deal with large conformational changes in the modeling of biomolecular complexes. Structure 19, 555-565. doi: 10.1016/j.str.2011.01.014

Keskin, O., Bahar, I., Badretdinov, A. Y., Ptitsyn, O. B., and Jernigan, R. L. (1998). Empirical solvent-mediated potentials hold for both intra-molecular and inter-molecular inter-residue interactions. Protein Sci. 7, 2578-2586. doi: $10.1002 /$ pro.5560071211

Keskin, O., Bahar, I., Flatow, D., Covell, D. G., and Jernigan, R. L. (2002a). Molecular mechanisms of chaperonin GroEL-GroES function. Biochemistry 41, 491-501. doi: 10.1021/bi011393x

Keskin, O., Durell, S. R., Bahar, I., Jernigan, R. L., and Covell, D. G. (2002b). Relating molecular flexibility to function: a case study of tubulin. Biophys. J. 83, 663-680. doi: 10.1016/S0006-3495(02)75199-0

Keskin, O., Jernigan, R. L., and Bahar, I. (2000). Proteins with similar architecture exhibit similar large-scale dynamic behavior. Biophys. J. 78, 2093-2106. doi: 10.1016/S0006-3495(00)76756-7

Kim, M. H., Seo, S., Jeong, J. I., Kim, B. J., Liu, W. K., Lim, B. S., et al. (2013). A mass weighted chemical elastic network model elucidates closed form domain motions in proteins. Protein Sci. 22, 605-613. doi: 10.1002/pro.2244

Martin, D. R., Ozkan, S. B., and Matyushov, D. V. (2012). Dissipative electro-elastic network model of protein electrostatics. Phys. Biol. 9:036004. doi: 10.1088/1478-3975/9/3/036004

May, E. R., and Brooks, C. L. III. (2011). Determination of viral capsid elastic properties from equilibrium thermal fluctuations. Phys. Rev. Lett. 106:188101. doi: 10.1103/PhysRevLett.106.188101

Murphy, B. R., and Webster, R. G. (2001). "Orthomyxoviruses," in Fields Virology, eds B. N. Fields, D. M. Knipe, P. M. Howley, and D. E. Griffini (Philadelphia, PA: Lippincott Williams \& Wilkins).
Peng, C., and Head-Gordon, T. (2011). The dynamical mechanism of autoinhibition of AMP-activated protein kinase. PLoS Comput. Biol. 7:e1002082. doi: 10.1371/journal.pcbi.1002082

Ruvinsky, A. M., Kirys, T., Tuzikov, A. V., and Vakser, I. A. (2012). Structure fluctuations and conformational changes in protein binding. J. Bioinform. Comput. Biol. 10:1241002. doi: 10.1142/S0219720012410028

Sanejouand, Y. H. (2013). Elastic network models: theoretical and empirical foundations. Methods Mol. Biol. 924, 601-616. doi: 10.1007/978-1-62703-017-5_23

Schueler-Furman, O., and Wodak, S. J. (2016). Computational approaches to investigating allostery. Curr. Opin. Struct. Biol. 41, 159-171. doi: 10.1016/j.sbi.2016.06.017

Sen, T. Z., Feng, Y., Garcia, J. V., Kloczkowski, A., and Jernigan, R. L. (2006). The extent of cooperativity of protein motions observed with elastic network models is similar for atomic and coarser-grained models. J. Chem. TheoryComput. 2, 696-704. doi: 10.1021/ct600060d

Skehel, J. J., and Wiley, D. C. (1998). Coiled coils in both intracellular vesicle and viral membrane fusion. Cell 95, 871-874. doi: 10.1016/S0092-8674(00)81710-9

Tama, F., and Sanejouand, Y. H. (2001). Conformational change of proteins arising from normal mode calculations. Protein Eng. 14, 1-6. doi: 10.1093/protein/14.1.1

Teodoro, M. L., Philips, G. N. Jr., and Kavraki, L. E. (2002). “A dimensionality reduction approach to modeling protein flexibility," in International Conference on Computational Molecular Biology (RECOMB), 299-308. doi: $10.1145 / 565196.565235$

Teodoro, M. L., Philips, G. N. Jr., and Kavraki, L. E. (2003). Understanding protein flexibility through dimensionality reduction. J. Comput. Biol. 10, 617-634. doi: $10.1089 / 10665270360688228$

Tirion, M. M. (1996). Large amplitude elastic motions in proteins from a single-parameter, atomic analysis. Phys. Rev. Lett. 77, 1905-1908. doi: 10.1103/PhysRevLett.77.1905

Tsai, C. J., and Nussinov, R. (2014). A unified view of "how allostery works." PLoS Comput. Biol. 10:e1003394. doi: 10.1371/journal.pcbi.1003394

Uyar, A., Kurkcuoglu, O., Nilsson, L., and Doruker, P. (2011). The elastic network model reveals a consistent picture on intrinsic functional dynamics of type II restriction endonucleases. Phys. Biol. 8:056001. doi: $10.1088 / 1478-3975 / 8 / 5 / 056001$

Wieninger, S. A., Serpersu, E. H., and Ullmann, G. M. (2011). ATP binding enables broad antibiotic selectivity of aminoglycoside phosphotransferase( $\left.3^{\prime}\right)$-IIIa: an elastic network analysis. J. Mol. Biol. 409, 450-465. doi: 10.1016/j.jmb.2011.03.061

Wild, C. T., Shugars, D. C., Greenwell, T. K., McDanal, C. B., and Matthews, T. J. (1994). Peptides corresponding to a predictive alpha-helical domain of human immunodeficiency virus type 1 gp41 are potent inhibitors of virus infection. Proc. Natl. Acad. Sci. U.S.A. 91, 9770-9774. doi: 10.1073/pnas.91.21. 9770

Wilson, I. A., Skehel, J. J., and Wiley, D. C. (1981). Structure of the haemagglutinin membrane glycoprotein of influenza virus at $3 \mathrm{~A}$ resolution. Nature 289, 366-373. doi: 10.1038/289366a0

Wodak, S. J., Paci, E., Dokholyan, N. V., Berezovsky, I. N., Horovitz, A., Li, J., et al. (2019). Allostery in its many disguises: from theory to applications. Structure 27, 566-578. doi: 10.1016/j.str.2019.01.003

Xu, R., and Wilson, I. A. (2011). Structural characterization of an early fusion intermediate of influenza virus hemagglutinin. J. Virol. 85, 5172-5182. doi: 10.1128/JVI.02430-10

Yang, L., Song, G., Carriquiry, A., and Jernigan, R. L. (2008). Close correspondence between the motions from principal component analysis of multiple HIV1 protease structures and elastic network modes. Structure 16, 321-330. doi: 10.1016/j.str.2007.12.011

Yang, L., Song, G., and Jernigan, R. L. (2007). How well can we understand largescale protein motions using normal modes of elastic network models? Biophys. J. 93, 920-929. doi: 10.1529/biophysj.106.095927

Yang, L., Song, G., and Jernigan, R. L. (2009). Protein elastic network models and the ranges of cooperativity. Proc. Natl. Acad. Sci. U.S.A. 106, 12347-12352. doi: 10.1073/pnas.0902159106

Zhang, Y., Doruker, P., Kaynak, B., Zhang, S., Krieger, J., Li, H., et al. (2020). Intrinsic dynamics is evolutionarily optimized to enable allosteric behavior. Curr. Opin. Struct. Biol. 62, 14-21. doi: 10.1016/j.sbi.2019.11.002 
Zheng, W. (2011). Accurate flexible fitting of high-resolution protein structures into cryo-electron microscopy maps using coarse-grained pseudoenergy minimization. Biophys. J. 100, 478-488. doi: 10.1016/j.bpj.2010. 12.3680

Zheng, W., and Auerbach, A. (2011). Decrypting the sequence of structural events during the gating transition of pentameric ligand-gated ion channels based on an interpolated elastic network model. PLoS Comput. Biol. 7:e1001046. doi: 10.1371/journal.pcbi.1001046

Zhu, F., and Hummer, G. (2010). Pore opening and closing of a pentameric ligand-gated ion channel. Proc. Natl. Acad. Sci. U.S.A. 107, 19814-19819. doi: 10.1073/pnas.1009313107

Zimmermann, M. T., Kloczkowski, A., and Jernigan, R. L. (2011b). MAVENs: motion analysis and visualization of elastic networks and structural ensembles. BMC Bioinformatics 12:264. doi: 10.1186/1471-2105-12-264

Zimmermann, M. T., Leelananda, S. P., Gniewek, P., Feng, Y., Jernigan, R. L., and Kloczkowski, A. (2011c). Free energies for coarse-grained proteins by integrating multibody statistical contact potentials with entropies from elastic network models. J. Struct. Funct. Genomics 12, 137-147. doi: $10.1007 /$ s10969-011-9113-3
Zimmermann, M. T., Leelananda, S. P., Kloczkowski, A., and Jernigan, R. L. (2012). Combining statistical potentials with dynamics-based entropies improves selection from protein decoys and docking poses. J. Phys. Chem. B 116, 6725-6731. doi: 10.1021/jp2120143

Zimmermann, M. T., Skliros, A., Kloczkowski, A., and Jernigan, R. L. (2011a). Immunoglobulin structure exhibits control over CDR motion. Immunome Res. 7:5. doi: 10.4172/1745-7580.1000047

Conflict of Interest: The authors declare that the research was conducted in the absence of any commercial or financial relationships that could be construed as a potential conflict of interest.

Copyright (C) 2021 Jernigan, Sankar, Jia, Faraggi and Kloczkowski. This is an openaccess article distributed under the terms of the Creative Commons Attribution License (CC BY). The use, distribution or reproduction in other forums is permitted, provided the original author(s) and the copyright owner(s) are credited and that the original publication in this journal is cited, in accordance with accepted academic practice. No use, distribution or reproduction is permitted which does not comply with these terms. 